CARTA AL EDITOR

\section{TELECONSULTA EN ONCOLOGÍA: EXPERIENCIA EN UN HOSPITAL DEL PERÚ DURANTE LA PANDEMIA}

\section{TELECONSULTATIONS IN ONCOLOGY: EXPE- RIENCE IN A HOSPITAL IN PERU DURING THE PANDEMIC}

\author{
Sally Rose Paredes-Noguni(i) 1,2,a, \\ Denisse Angélica Castro-Uriol(101,2,a, \\ Renzo Mauricio Salas-Rojas (101,a, Percy Soto-Becerra (103,b, \\ Brady Ernesto Beltrán-Gárate (1) 1,2,a

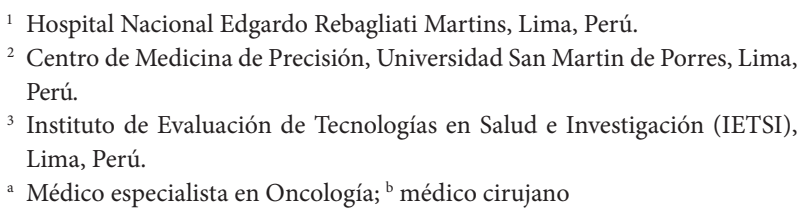

Sr. Editor: El 11 de marzo de 2020, la Organización Mundial de la Salud (OMS) declaró la pandemia por COVID-19 debido al elevado número de casos reportados fuera de China. El Perú inició un periodo de aislamiento social obligatorio el 15 de marzo, tan solo 11 días después del primer caso reportado ${ }^{(1)}$. En el contexto actual, la telesalud ha brindado una solución al restablecimiento de la atención en salud a través de la teleconsulta y el telemonitoreo, donde, pese a que el Perú ya contaba con una Ley Marco de Telesalud que optimizaba los procesos vinculados a su desarrollo, tales como la teleconsulta ${ }^{(2)}$; durante la pandemia del COVID-19 se establecieron algunas recomendaciones internacionales ${ }^{(3)} \mathrm{y}$ se actualizaron las normas nacionales existentes para su implementación ${ }^{(4)}$.

Específicamente, el uso de la telemedicina aplicada al paciente oncológico se denomina teleoncología y numerosas sociedades oncológicas, tales como la americana (ASCO) y la inglesa (NIH), están adoptando su utilización y sugerido su uso internacional con miras a reducir las brechas de atención entre países desarrollados y en vías de desarrollo ${ }^{(5,6)}$. Aprovechando la pertinencia de la teleoncología en tiempos del COVID-19, el Servicio de Oncología del Hospital Nacional Edgardo Rebagliati Martins (HNERM) y el Centro Nacional de Telemedicina de EsSalud Seguro Social de Salud del Perú (CENATE) diseñaron e implementaron una intervención de

\footnotetext{
Citar como: Paredes-Noguni SR, Castro-Uriol DA, Salas-Rojas RM, Soto-Becerra P, BE Beltrán-Gárate. Teleconsultas en oncología: experiencia en un hospital del Perú durante la pandemia. Rev Peru Med Exp Salud Publica. 2021;38(1):178-9. doi: https://doi.org/10.17843/rpmesp.2021.381.6237.
}

Correspondencia: Sally Rose Paredes-Noguni; sallyparedeslggg@gmail.com telemedicina basada en teleconsultas y telemonitoreo con la finalidad de restablecer prontamente la atención médica a los pacientes oncológicos, cuya seguridad depende fundamentalmente de un oportuno manejo y permanente monitoreo (Anexo 1). En esta carta describimos la implementación de la intervención de teleconsultas en oncología.

Para esta intervención, oncólogos médicos realizaron diariamente una selección entre los pacientes originalmente programados para dicho día en los 14 turnos de consulta externa dispuestos previamente. Se realizó un triaje para definir a los pacientes que califican para teleconsulta, los cuales son idealmente pacientes con hormonoterapia profiláctica por cáncer de mama in situ o de intención adyuvante por cáncer de mama localizado y cáncer de próstata no metastásico, tributarios de tratamiento oral e/o intramuscular. Esto basado en que son pacientes con una tendencia a una indicación prolongada de medicación y en los cuales no requerimos estrictamente exámenes auxiliares de laboratorio para cada consulta. En estos pacientes se realiza contacto telefónico en las 24 horas previas para corroborar la aceptación verbal de la teleconsulta y realizar capacitación sobre el uso del aplicativo Zoom, con el apoyo de CENATE (Centro Nacional de Telemedicina de EsSalud); con ello, se elabora la lista final de pacientes confirmados, a quienes se le es asignada la cita, la cual se efectiviza teniendo al paciente en su domicilio y se toma registro en la historia clínica física y digitalizada del paciente, siendo emitida la receta única estandarizada, la cual es entregada a un módulo que concreta la llegada del medicamento al familiar del paciente. Durante la consulta, nuevamente se ratifica el consentimiento verbal del paciente a la ejecución de esta bajo esta modalidad, lo cual se consigna en la hoja de atención digital.

Se realizó una prueba piloto que contó con la participación de un médico oncólogo y cuatro pacientes. Desde el día 25 de marzo del 2020 se inició la teleconsulta, entre esta fecha y el 20 de abril se atendieron 344 pacientes en 21 días de un total de 554 pacientes originalmente seleccionados durante el triaje (62,1\% de teleconsultas concretizadas). La edad media de los pacientes atendidos fue de 64,3 años (desviación estándar de 11,6) y varió entre 33 a 93 años. En promedio, hubo 16 teleconsultas por día (desviación estándar de 3, rango de 11 a 22). La mayoría de teleconsultas fue a pacientes con cáncer de mama $(68,3 \%, 235 / 344)$ seguido de pacientes con cáncer de próstata $(29,4 \%, 101 / 344)$. Los restantes fueron tres por cáncer de útero, dos por cáncer de recto, dos por cáncer de colon y uno por cáncer de estómago. La Figura 1 muestra la evolución durante el primer mes de realizadas las teleconsultas.

Uno de los principales retos de esta intervención es el desconocimiento por parte de la población de aplicativos en internet específicos para la teleconsulta, la calidad de la red a nivel bilateral, asimismo el poco tiempo que se ha empleado para la implementación. Esto último si bien puede plantear- 


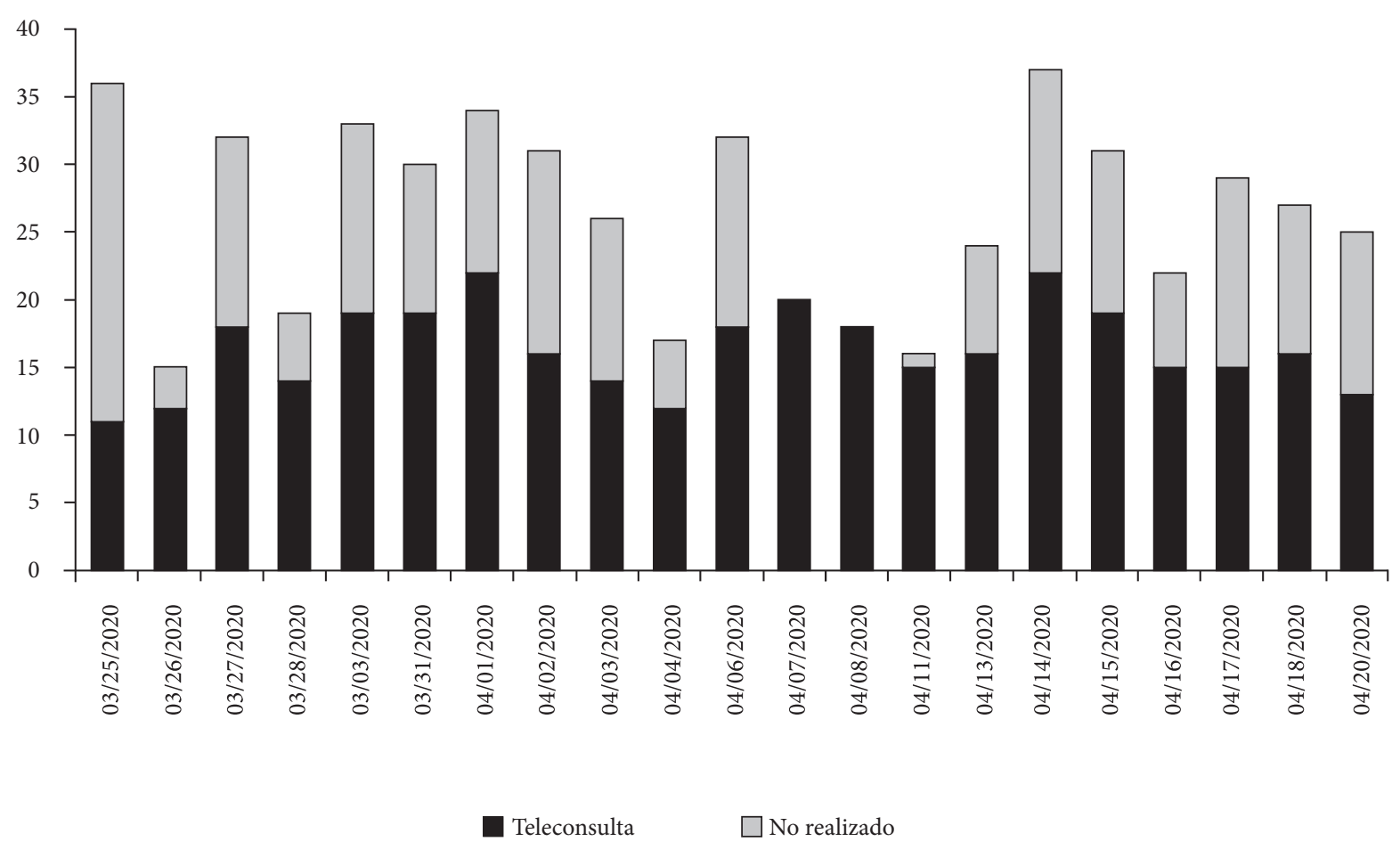

Figura 1. Teleconsultas en oncología realizadas en un hospital del seguro social del Perú

se como una limitación, constituye al mismo tiempo una oportunidad de mejora para el servicio y la institución. Al momento, la evaluación de los procesos y la satisfacción del usuario interno y externo se encuentra en desarrollo.

En conclusión, los primeros resultados en la implementación de esta intervención en el Servicio de Oncología Médica del Hospital Nacional Edgardo Rebagliati Martins, demuestran que es posible iniciar con las teleconsultas, aunque la intervención fue desarrollada para cubrir necesidades de accesibilidad a consultas médicas y continuidad de tratamiento durante la pandemia; el alcance puede ser mayor y se constituiría en una valiosa herramienta institucional, al permitir descongestionar el saturado sistema de citas hospitalarias.

Contribuciones de los autores: SRPN, RSR y BBG han participado en la concepción y diseño de la intervención. SRPN y DCU han participado en la recolección de resultados y redacción del artículo. PSB ha participado en el análisis e interpretación de los datos.

Financiamiento: Autofinanciado.

Conflictos de interés: Los autores declaran no tener conflictos de interés.

Material suplementario: Disponible en la versión electrónica de la RPMESP.

\section{REFERENCIAS BIBLIOGRÁFICAS}

1. El Peruano. Decreto Supremo que declara Estado de Emergencia Nacional por las graves circunstancias que afectan la vida de la Nación a consecuencia del brote del COVID-19 - Decreto Supremo 044-2020-PCM [Internet]. Disponible en:https://busquedas.elperuano.pe/normaslegales/ decreto-supremo-que-declara-estado-de-emergencia-nacional-po-decreto-supremo-n-044-2020-pcm-1864948-2/.

2. Presidencia de la República. Decreto Supremo 003-2019-SA. Ley Marco de Telesalud modificada con el Decreto Legislativo 1303, Decreto Legislativo que optimiza procesos vinculados a Telesalud. [Internet]. Disponible en: https:// busquedas.elperuano.pe/download/url/aprueban-el-reglamento-de-la-leyn-30421-ley-marco-de-tele-decreto-supremo-n-003-2019-sa-1741932-4.

3. Portnoy J, Waller M, Elliott T. Telemedicine in the Era of COVID-19. J Allergy Clin Immunol Pract. 2020;8(5):1489-1491. doi: 10.1016/j. jaip.2020.03.008.

4. El Peruano. Aprueban la Directiva Administrativa $N^{\circ}$ 286-MINSA/2020/ DIGTEL: Directiva Administrativa para la Implementación y Desarrollo de los Servicios de Teleorientación y Telemonitoreo - Resolucion MinisteriaL 0146-2020-MINSA. [Internet]. Disponible en: https://busquedas.elperuano. pe/normaslegales/aprueban-la-directiva-administrativa-n-286-minsa2020digte-resolucion-ministerial-n-0146-2020-minsa-1865268-6/.

5. Ngwa W, Olver I, Schmeler KM. The Use of Health-Related Technology to Reduce the Gap Between Developed and Undeveloped Regions Around the Globe. Am Soc Clin Oncol Educ Book. 2020;40:1-10. doi:10.1200/EDBK_288613.

6. Sirintrapun SJ, Lopez AM. Telemedicine in Cancer Care. Am Soc Clin Oncol Educ B. 2018;(38):540-5. doi: 10.1200/EDBK_200141. 